\title{
Importância das manifestações não motoras da Doença de Parkinson
}

\author{
Importance of the non-motor manifestations of the Parkinson's disease.
}

\author{
Egberto Reis Barbosa', Luciano Magalhães Melo²
}

\section{RESUMO}

A doença de Parkinson (DP) é considerada essencialmente doença motora caracterizada por tremor de repouso, rigidez, bradicinesia, e distúrbios posturais. Entretanto, complicações não motoras, incluindo distúrbios do humor, ansiedade, fadiga, apatia, psicose, demência, distúrbios do sono e disfunção autonômica freqüentemente estão presentes nesta moléstia e têm impacto negativo na qualidade de vida dos pacientes e de seus cuidadores. O processo degenerativo próprio da DP, drogas utilizadas no tratamento da DP e reações emocionais frente à doença crônica, são as causas desses sintomas. Nesta revisão serão abordados as principais manifestações não motoras da DP.

Unitermos: Doença de Parkinson, Demência, Transtornos Psicóticos, Distúrbios do sono.

Citação: Barbosa ER, Melo LM. Importância das manifestações não motoras da Doença de Parkinson. Rev Neurocienc 2007;15(1):49-59.

\section{SUMMARY}

Parkinson's disease (PD) is primarily considered a motor disease characterized by rest tremor, rigidity, bradykinesia and postural disturbances. However, non-motor complications, including mood and anxiety disorders, fatigue, apathy, psychosis, dementia, sleep disorders and autonomic dysfunction frequently are present in PD and have a substantial negative impact on quality of life of the patients and their caregivers. Neurobiological changes of this degenerative disease, drugs for its treatment and emotional reactions to a disabling chronic disease probably are the most important cause of those symptoms. In this review the main non-motor manifestations PD are discussed.

\section{Keywords: Parkinson Disease, Dementia, Psychotic Disorders, Sleep disorder.}

Citation: Barbosa ER, Melo LM. Importance of the non-motor manifestations of the Parkinson's disease. Rev Neurocienc 2007;15(1):49-59.

\section{INTRODUÇÃO}

Pode-se dividir as manifestações não motoras da DP em autonômicas (ex: obstipação intestinal, hipotensão ortostática), alterações sensoriais (dores de diversos tipos), em neuropsiquiátricas (ex: demência e depressão) e em distúrbios do sono. É importante frisar que o próprio tratamento da DP pode trazer complicações não motoras. As manifestações não motoras da doença de DP embora por vezes relegadas a um se- gundo plano de importância têm considerável impacto na qualidade de vida dos portadores da moléstia ${ }^{1,2}$. Weintraub et $\mathrm{al}^{2}$ ressaltam a necessidade de que se pesquise sistematicamente a presença de sintomas não motores em indivíduos com DP, a fim de avaliar de maneira mais abrangente as limitações ocasionadas pela moléstia. Em resposta a essa necessidade, foi validado pelo Multidisciplinary International Parkinson's Disease Non Motor Group, o primeiro questionário ${ }^{3}$ para

Trabalho realizado no Departamento de Neurologia da Faculdade de Medicina da Universidade de São Paulo.

1.Professor Livre Docente pelo Departamento de Neurologia da Faculdade de Medicina da Universidade de São Paulo. Coordenador do Grupo de Estudo de Distúrbios do Movimento da Clínica Neurológica do Hospital das Clínicas da Faculdade de Medicina da Universidade de São Paulo

2.Médico Neurologista Colaborador do Grupo de Estudo de Distúrbios do Movimento da Clínica Neurológica do Hospital das Clínicas da Faculdade de Medicina da Universidade de São Paulo 
acessar e quantificar a importância que traz os sintomas não motores na DP. Este questionário de em 30 itens, abrange os seguintes sintomas não motores da DP: capacidade de conter salivação, perda de olfato e perda de paladar, disfagia, constipação, incontinência fecal, sensação de esvaziamento incompleto de intestino, urgência miccional, noctúria, dores inexplicáveis, perda inexplicável de peso, redução de memória, sintomas depressivos, alucinações, redução de capacidade de se concentrar, ansiedade, sensação de medo ou pânico, distúrbios sexuais, hipotensão postural, quedas, sonolência, parassonias, edema de membros inferiores e suor excessivo.

Em estudo de um grupo de 99 pacientes com DP, Shulman et $\mathrm{al}^{4}$ constataram que $88 \%$ dos pacientes apresentavam pelo menos uma manifestação não motora da moléstia e $11 \%$ dos casos apresentavam cinco manifestações não motoras, sendo que os itens incluídos foram: ansiedade, depressão, distúrbios sensoriais, fadiga e alterações do sono. No presente artigo de revisão serão abordadas as manifestações não motoras mais importantes da DP e complicações do tratamento também não motoras.

\section{Demência}

A presença de alterações cognitivas na DP foi ignorada por muito tempo. Porém, sabe-se que déficits cognitivos eventualmente ocorrem já nas fases iniciais da DP, sendo que nessas circunstâncias podem não ser clinicamente aparentes, mas detectáveis apenas por testes específicos.

O termo demência associada à DP refere-se à demência que se desenvolve pelo menos doze meses após a instalação das alterações motoras. Quando a demência surge já nos primeiros doze meses de evolução do quadro motor caracteriza-se quadro clínico da demência de corpos de Lewy 5 .

Dentre as alterações cognitivas presentes na DP, a demência associada à DP é a manifestação mais grave e a sua presença reduz a sobrevida dos pacientes ${ }^{6}$. A demência associada à DP, assim como outras manifestações neuropsiquiátricas associadas a essa moléstia, também acarreta redução da qualidade de vida dos pacientes e até mesmo de seus cuidadores ${ }^{7}$. Além disso, a demência associada à DP é muito comum, com prevalência estimada por alguns estudos variando entre 20 a 40\%1,8 e prevalência acumulada podendo chegar a $80 \%$ de acordo com os resultados do estudo de Aarsland et $a^{9}{ }^{9}$. Vale ressalvar que a média da idade da população examinada neste estudo era superior a 70 anos, o que pode ter contribuído para encontrar prevalência tão alta.

Aarsland et $\mathrm{a}^{10}$ demonstraram que outras manifestações neuropsiquiátricas podem estar associadas à demência da DP. Neste estudo os autores mostraram que $89 \%$ de 537 pacientes com demência associada à DP apresentavam pelo menos um outro sintoma neuropsiquiátrico e $77 \%$ sofreram dois ou mais sintomas. Os sintomas mais comuns foram: depressão (58\%), apatia (54\%), ansiedade (49\%) e alucinações (44\%). Os pacientes com doença mais grave e depressão mais acentuada sofreram maior número de sintomas neuropsiquiátricos associados. De acordo com dados revelados por este mesmo estudo, aproximadamente $60 \%$ dos cuidadores apresentavam pelo menos um sintoma neuropsiquiátrico. Aqueles enfermos com agitação e psicose obtiveram as menores pontuações no MiniExame do Estado Mental e maiores na escala UPDRS, e os seus cuidadores apresentavam os piores níveis de estresse. É importante notar a importância da apatia na DP, como observado neste estudo. A apatia está mais relacionada às alterações biológicas da doença do que à reação psicológica à moléstia. Esta manifestação está intimamente associada à perda cognitiva ${ }^{11}$ e não à depressão, de acordo com os dados de Kirsch-Darrow et $\mathrm{al}^{12} \mathrm{em}$ estudo que comparou apatia em 80 pacientes com DP e 20 com distonia. Os autores verificaram que apatia foi mais comum nos parkinsonianos e é independente de depressão.

O risco de desenvolver demência é duas vezes maior em indivíduos com DP que os controles da mesma ida$\mathrm{de}^{13}$. Os fatores de risco mais relevantes para demência em DP são6,9,14-18: 1) idade avançada; 2) pontuação na escala de Hoehn \& Yahr superior a dois pontos; 3) pontuação no Mini-Exame do Estado Mental inferior a 29; 4) disfunção cognitiva em domínios que não memória ou em múltiplos domínios cognitivos; 5) sinais de disfunção do lobo frontal; 6) Parkinsonismo com predomínio de bradicinesia ou padrão misto: tremor e bradicinesia; 7) baixo nível educacional; 8) ser portador de ao menos um alelo e4 do gene apolipoproteina E (APOE e4) e especialmente, do alelo e2 (APOE e2); 9) presença de transtorno cognitivo leve; 10) presença de alucinações induzidas por agentes dopamingérgicos.

Distúrbios visuoespaciais e lentificação de processos decisivos são alterações cognitivas isoladas que podem surgir precocemente na evolução da DP sem que representem à instalação de um quadro demencial. Diferentemente, o quadro demencial na DP instala-se em fases mais adiantadas da evolução da moléstia e tem como principais características a lentificação do processo cognitivo (bradifrenia), a apatia, o comprometimento da memória e das funções executivas frontais ${ }^{19}$.

A identificação de alterações cognitivas na DP oferece algumas dificuldades. As funções executivas, que representam os domínios cognitivos geralmente afetados na DP, habitualmente não são avaliadas em testes de rotina. São, portanto, necessários testes específicos para avaliar essas funções que compreendem: formação de conceitos; solução de problemas; capacidade 
(aptidão) para mudança de padrões; e elaboração de estratégias.

De acordo com as mais recentes recomendações da Academia Americana de Neurologia, baseadas na metanálise de Miyasaki et a ${ }^{20}$ o Mini-Exame do Estado Mental e o Exame Cognitivo de Cambridge (Cambridge Cognitive Examination, CAMCog) são recursos úteis para rastreamento de demência em pacientes com DP. Note-se que ambos têm sensibilidade equiparável, mas o CAMCog é mais específico por ser mais abrangente, embora requeira tempo maior para sua aplicação. Apesar de ainda não serem muito utilizados e de estarem fora das recomendações da Academia Americana de Neurologia, duas outras escalas foram criadas especialmente para serem aplicadas em indivíduos com DP: a Escala para Resultados de Cognição na Doença de Parkinson (Scales for Outcomes of Parkinson's Disease-Cognition, SCOPA-COG) ${ }^{21}$ e o teste Mini-Mental Parkinson ${ }^{22}$. Essas escalas foram elaboradas com devida atenção às peculiaridades das perdas cognitivas características da DP.

A diferenciação da demência da DP deve ser feita em relação às seguintes condições: depressão, confusão mental, demência dos corpos de Lewy e hidrocefalia de pressão normal ${ }^{23}$.

Uma importante conseqüência da demência na DP é a restrição quanto ao uso de drogas antiparkinsonianas, desde que nessas circunstâncias essas drogas são muito mais propensas a provocarem efeitos colaterais neuropsiquiátricos. A implicação clínica decorrente é o controle precário das dificuldades motoras e uma redução da sobrevida. Dessa forma o manejo do quadro demencial na DP envolve uma rigorosa seleção dos antiparkinsonianos a serem empregados, evitando-se o uso principalmente de fármacos com ação anticolinérgica.

Estudos a respeito do efeito de drogas de ação colinérgica tais como a rivastigmina, o donezepil e a galantamina, freqüentemente empregados no tratamento da doença de Alzheimer, sobre as alterações cognitivas da DP mostraram resultados favoráveis, sem piora do quadro motor ${ }^{24-26}$. É importante mencionar que a rivastigmina foi aprovada pelo FDA e pela ANVISA para o tratamento da demência associada à DP. De acordo com as recomendações da Academia Americana de Neurologia a rivastigmina pode ser usada para o tratamento de demência associada à DP, e o donepezil, pode ser prescrito com mesma finalidade ${ }^{20}$.

Ao se avaliar um paciente com DP que apresenta alterações cognitivas, é importante descartar a presença de alterações metabólicas e efeitos tóxicos de drogas. Pioras bruscas podem ter causas de natureza não degenerativa, como infecção e hematoma subdural. Depressão também deve ser afastada, pois essa condição pode piorar o desempenho cognitivo.
Em estudo recente, multicêntrico, duplo-cego, placebo-controlado, Emre et $\mathrm{a}^{27}$ avaliaram o efeito da rivastigmina (dose de 3 a12 mg) em 541 pacientes com DP que apresentavam quadro demencial leve ou moderado. O período de seguimento foi de 24 semanas e os autores observaram que os pacientes que receberam rivastigmina comparados com o quadro placebo apresentaram melhora moderada do quadro demencial, porém neste grupo houve maior incidência de efeitos colaterais, dos quais os mais importantes foram: náuseas (29 \% no grupo ativo e 11,2 \%no grupo placebo), vômitos (16,6 no grupo ativo e 1,7 \% no grupo placebo) e tremor (10,2 no grupo ativo e 3,9 \% no grupo placebo). Apesar da maior ocorrência de tremor no grupo recebendo rivastigmina não foi constatada diferença significativa nos escores motores da UPDRS entre o grupo placebo e o grupo ativo. Uma extensão deste estudo foi recentemente publicada por Poewe et al ${ }^{28}$, que demonstraram que os efeitos benéficos da terapia com rivastigmina prolongam-se por mais 24 semanas.

\section{Depressão}

A depressão é sem dúvida o distúrbio neuropsiquiátrico mais comum na DP. Sua prevalência varia bastante (4\% - $70 \%$ ) nos diferentes estudos, a depender da metodologia empregada, porém, situa-se em torno de $40 \%$ em pesquisas recentes que utilizaram escalas de avaliação mais adequadas como a de Beck ou a de Hamilton29.

Não há uma correlação bem estabelecida entre depressão e a idade atual do paciente ou a idade do paciente no início da $\mathrm{DP}^{30}$, embora Starkstein et al ${ }^{31}$ tenham observado que a depressão foi mais comum e mais grave em parkinsonianos em que a doença instalou-se mais precocemente (antes de 55 anos de idade). Não há relação também entre os sintomas depressivos e a duração da doença, como seria esperado. Vários estudos demonstraram maior incidência de depressão entre pacientes de sexo feminino com DP e indivíduos com história pessoal ou familiar de depressão, têm mais chance de tornarem-se deprimidos após o início da $\mathrm{DP}^{32}$.

A depressão parece ser mais comum em pacientes parkinsonianos em que predominam rigidez e bradicinesia, do que naqueles que apresentam tremor como sintomatologia principal ${ }^{31}$. Alguns estudos sugerem ainda que os pacientes com hemiparkinsonismo direito (disfunção maior no hemisfério cerebral esquerdo), obtém escores mais altos na escala de avaliação de Beck, do que aqueles com predominância de sinais no dimidio esquerdo ou envolvimento bilateral ${ }^{33}$.

A depressão na DP é caracterizada pelo alto nível de ansiedade, pessimismo sobre o futuro, tristeza, irritabilidade, preocupação excessiva com saúde, ideação 
suicida, porém com baixa incidência de suicídio, e, uma falta relativa de alucinações, idéias delirantes, sentimento de culpa e autocrítica excessiva. Cerca de metade dos pacientes deprimidos com DP, se enquadram nos critérios de depressão maior, enquanto a outra metade apresenta distimia ou depressão leve a moderada ${ }^{34}$.

Há controvérsias sobre as causas da depressão na DP. Seria um processo reativo a uma enfermidade crônica ou mais uma manifestação das anormalidades neurobiológicas presentes nessa doença ${ }^{29}$. Os seguintes argumentos favorecem a segunda hipótese: a) freqüentemente a depressão manifesta-se antes que os sinais e sintomas motores se instalem, podendo preceder o quadro motor em até vários anos; b) a depressão é mais prevalente em pacientes com DP do que em indivíduos portadores de doenças crônicas igualmente incapacitantes; c) a depressão não está necessariamente correlacionada diretamente com a gravidade da doença; d) a depressão na DP tem características próprias (descritas acima).

Algumas características inerentes à DP, contribuem para dificultar o diagnóstico da depressão associada à DP. Alguns sintomas são comuns à depressão e à DP, logo um indivíduo parkinsoniano com apatia, insônia ou sono excessivo, com perda de peso, talvez não sofra necessariamente de depressão. Um paciente com face congelada, voz baixa, lentificado, e com postura curvada pode parecer deprimido, mas muitas vezes não está. A depressão pode agravar a lentidão dos pacientes, sem que esses atribuam tais manifestações a outro problema que não diretamente a $\mathrm{DP}^{35}$. Face às essas dificuldades, uma história clínica cuidadosa, exame físico detalhado e o emprego de questionários específicos, podem auxiliar no diagnóstico. As escalas recomendadas pela Academia Americana de Neurologia, baseadas na metanálise de Miyasak et al ${ }^{20}$ para avaliar a presença de depressão na DP são: A escala BDI (Beck Depression Inventory), e a escala HDRS (Hamilton Depression Rating Scale). A escala MADRS (Montgomery Asberg Depression Rating Scale) é possivelmente útil para triagem de depressão associada à DP.

Das drogas comumente utilizadas no tratamento da DP, a selegilina tem efeito antidepressivo, ainda que modesto. A levodopa, embora possa melhorar a apatia e a perda de motivação associadas à disfunção da via dopaminérgicas meso-fronto-límbica, eventualmente pode ter efeito depressogênico ${ }^{36}$. Por outro lado há dados indicativos de que o pramipexol tem efeito positivo sobre o humor nos parkinsonianos, conforme estudo recente de Barone et $\mathrm{al}^{37}$. Neste estudo os autores conduziram um ensaio randomizado, aberto, com duração de 14 semanas com o objetivo de comparar o efeito sobre a depressão em pacientes com DP de 2 drogas: a sertralina (dose de 50 mg/dia), droga antidepresiva de efeito já comprovado e o agonista dopaminérgico de efeito antiparkinsoniano pramipexol (dose de 1,5 a
4,5 mg/dia). Participaram do ensaio 7 centros italianos e nos quais foram recrutados 67 pacientes com DP e depressão maior mas sem flutuações motoras ou discinesias. A escalas utilizadas para a avaliação dos pacientes foram a Hamilton Depression Rating Scale (HDRS) para depressão e a Unified Parkinson's Disease Rating Scale (UPDRS) para aferição das manifestações motoras da DP. Os pacientes foram considerados recuperados da depressão quando apresentavam escore na HAM-D igual ou inferior a 8 pontos. Os resultados foram até certo ponto surpreendentes, pois embora ambos os tratamentos tenham mostrado queda no escore da HAM-D a porcentagem de pacientes recuperados com o pramipexol $(60,6 \%)$ foi significativamente superior à dos recuperados com a sertralina (27,3\%).

Em relação ao tratamento convencional da depressão na DP, embora existam poucos estudos bem controlados direcionados a esta questão, as diferentes classes de antidepressivos, podem ter efeito antidepressivo na DP, incluindo-se os antidepressivos tricíclicos e os inibidores seletivos da recaptação da serotonina (ISRS): fluoxetina, paroxetina, sertralina, citalopram e fluvoxamina ${ }^{38}$. Porém, antidepressivos da classe dos ISRS podem agravar o parkinsonismo ${ }^{39}$. Deve-se considerar ainda que pacientes em uso da selegilina a introdução dos antidepressivos acima mencionados implica em risco, ainda que remoto, de desencadeamento da síndrome serotoninérgica, que se caracteriza por confusão mental associada a graves distúrbios autonômicos. A Academia Americana de Neurologia não recomenda antidepressivo especifico ou outro tipo de tratamento para a depressão associada à DP, embora aponte que as maiores evidências de eficácia são favoráveis a amitriptilina ${ }^{20}$.

A depressão da DP responde bem à eletroconvulsoterapia, observando-se ainda melhora transitória dos sintomas motores ${ }^{40}$. Essa modalidade de tratamento, entretanto, deve ser reservada a casos de depressão grave associada à DP, resistente à terapêutica farmacológica. A estimulação magnética transcraniana (EMT) tem mostrado resultados positivos para tratamento da depressão primária. Em pacientes com DP e depressão, Fregni et $\mathrm{al}^{41}$, em estudo comparando EMT e fluoxetina, observaram resposta positiva e equivalente com as duas modalidades de tratamento.

Não há estudos conclusivos sobre o efeito de tratamento cirúrgico da DP, sobre o humor dos pacientes, contudo, são preocupantes os relatos recentes de depressão grave e suicídio após a implantação de eletrodos em estruturas dos núcleos da base para estimulação cerebral profunda. Assim, Voon et $\mathrm{al}^{42}$ observaram 2 suicídios $(0,5 \%)$ e 7 tentativas $(1,7 \%)$ em 406 pacientes submetidos à estimulação do núcleo subtalâmico. Da mesma forma, Burckard et $\mathrm{al}^{43}$ constataram 5 suicídios (4 pacientes com DP e 1 com distonia) em 134 pacientes (3,7\%) submetidos à estimulação cerebral profunda para tratamento de distúrbios do movimento. 


\section{Fadiga}

Assim como outras manifestações não motoras da DP a fadiga é um sintoma pouco reconhecido pelos médicos. Esta tem incidência calculada entre $40 \%$ a $56 \%$ na PD e tem impacto negativo na qualidade de vida. Martinez-Martin et al ${ }^{44}$, demonstram associação entre fadiga e depressão, enquanto que Alves et al ${ }^{45}$, sugerem que a fadiga pode ser sintoma independente na DP, embora esteja presente como comorbidade conjunta com outras manifestações não motoras da DP. Herlofson e Larse46 demonstraram que a presença de fadiga se correlaciona com pior qualidade de vida e que parkinsonianos com fadiga tendem a ter maior comprometimento motor. Lou et a $\mathrm{l}^{47}$ demonstraram que pacientes com DP têm manifestações de fadiga em cinco modalidades: fadiga geral, fadiga física, motivação reduzida, atividade reduzida e fadiga mental.

Pouco se sabe sobre o tratamento da fadiga, mas Martinez-Martin et al ${ }^{44}$ observaram incidência reduzida de fadiga naqueles que estavam recebendo amantadina. Os agonistas dopaminérgicos foram testados contra a fadiga e não apresentaram efeito sobre esse sintoma assim como não se observou correlação entre sonolência causada por introdução de agonista dopaminérgico e piora de fadiga ${ }^{48}$. Por sua vez a levodopa parece reduzir fadiga, como verificado por Lou et al ${ }^{49}$, em estudo que focalizou apenas a fadiga física.

\section{Complicações não motoras decorrentes da terapia antiparkinsoniana.}

As alucinações visuais constituem o tipo mais comum de efeito colateral neuropsiquiátrico das drogas antiparkinsonianas, ocorrendo com freqüência que varia entre 25 e 40\% ${ }^{50}$. O estudo de Fenelon et al ${ }^{50}$ mostrou que apenas $12 \%$ dos pacientes referiam espontaneamente alucinações sendo que os demais não relatavam essas ocorrências por apresentarem comprometimento cognitivo significativo ou por receio de serem considerados insanos. Portanto esse tipo de efeito colateral deve ser investigado ativamente pelo médico através de perguntas feitas aos pacientes ou aos cuidadores. Estudos longitudinais mostram ainda que numa coorte de pacientes a prevalência das alucinações aumenta à medida que o tempo passa podendo atingir cerca de $50 \%$ dos casos ${ }^{51}$.

Embora o termo alucinação associada à DP seja freqüentemente utilizado para descrever sintomas relacionados a esta enfermidade o uso é inapropriado na maioria das vezes, pois os pacientes geralmente mantém a crítica frente a tais manifestações, percebendo sua natureza irreal e desta forma, o termo alucinose seria mais adequado.

Tipicamente os pacientes descrevem as manifestações alucinatórias como imagens bem estruturadas, silenciosas de pessoas ou animais. São percebidas enquanto os pacientes estão alerta e com os olhos abertos, surgem à noite ou no final do dia e persistem por segundos ou minutos. A crítica comumente permanece intacta. As imagens, geralmente mal definidas, aparecem sem nenhum fator desencadeante e comumente movem-se e desaparecem subitamente. Acredita-se que possa haver uma 2 formas de alucinações. A primeira teria início precoce (inferior a 5,5 anos após inicio dos sintomas) associada a flutuações motoras e doses altas de medicação, e a outra associada ao declínio cognitivo ${ }^{52}$. Quando as alucinações surgem em doentes com critica preservada, como regra, não causam medo, pois os pacientes mantêm sua percepção de que o que foi visto não é real. Porém alguns pacientes podem ficar amedrontados quando a percepção do realidade está comprometida.

Alucinações em outras modalidades sensoriais, como audição, tato, olfato e gustação são incomuns. As alucinações por ação de anticolinérgicos podem ser diferenciadas daquelas que surgem por ação de drogas dopaminérgicas. Estas ocorrem em geral com crítica preservada, enquanto que aquelas surgem freqüentemente no contexto de um quadro confusional. Outra peculiaridade das alucinações precipitadas por anticolinérgicos é que são mal estruturadas e por vezes ameaçadoras.

Os delírios são menos comuns que as alucinações e ocorrem com freqüência que varia de 3 a 17\%. São tipicamente paranóides ou persecutórios e freqüentemente ocorrem em pacientes que já apresentavam alucinações. Todas as modalidades de drogas antiparkinsonianas podem provocar delírios, porém os agonistas dopaminérgicos e a levodopa são mais freqüentemente associados a esse tipo de manifestação psiquiátrica, por vezes denominado psicose levodopa-induzida ${ }^{53}$.

Os fatores de risco mais relevantes para distúrbios psicóticos induzidos por drogas antiparkinsonianas são: idade avançada, distúrbio visual, duração da doença e sua gravidade, disfunção cognitiva, depressão, distúrbio do sono, história pregressa de doença psiquiátrica e exposição a esses medicamentos.

Embora doses elevadas de levodopa e outras drogas antiparkinsonianas tenham sido associadas a alucinações em indivíduos com DP suscetíveis, e que a diminuição de dose de agentes dopaminérgicos possa fazer desaparecerem as alucinações ou ao menos reduzi-las, uma relação direta causa-efeito entre droga especifica e alucinação permanece controversa. Por outro lado alguns estudos não demonstraram associação entre uso e dose de drogas dopaminérgicas com risco de desenvolver demência ${ }^{16,26}$.

Goetz et al ${ }^{54}$ administraram levodopa em altas doses em 5 pacientes com DP não demenciados que referiam alucinações diariamente e nenhum deles apresentou 
sintoma psicótico. Sanchez-Ramos et a| ${ }^{55}$ demonstraram que alucinações vividas por pacientes com DP tipicamente iniciam-se após 10 anos de tratamento com levodopa, são diretamente influenciadas pela dose da terapia e estão associadas a distúrbios do sono como sonhos vívidos despertares noturnos.

Em revisão recente Fenelon et $a^{156}$ demonstraram que transtornos psicóticos associados à DP podem fazer parte da história natural da moléstia, especialmente em contexto de depressão ou síndrome demencial.

Ao avaliar paciente com sintomas psicóticos é importante afastar doenças secundárias como uma simples infecção urinária, e drogas que podem provocar os sintomas e que não fazem parte do tratamento da DP, como por exemplo, antidepressivos tricíclicos. Realizada essas considerações, deverão ser retiradas drogas antiparkinsonianas na seqüência: anticolinérgicos, amantadina, selegelina e agonistas dopaminérgicos. Então redução da dose de levodopa e, de inibidores da enzima catechol-O-metiltransferase (COMT) poderá ser considerada, observando-se que a adição de inibidores da COMT, enzima que metaboliza a levodopa no sangue, pode predispor a aparição de alucinações, por apenas aumentar a biodisponibilidade da levodopa, pois drogas desta classe não causam diretamente alucinações ${ }^{57}$. Em alguns casos devera ser admitido o emprego de neurolépticos atípicos, com baixa afinidade por receptores D1 e D2, como a clozapina que tem alta afinidade por receptores $\mathrm{D} 4^{58}$. O uso da clozapina em doses de 25 a $50 \mathrm{mg}$ por dia, é o mais efetivo entre os neurolépticos no controle dos referidos transtornos neuropsiquiátricos e o único que não piora o parkinsonis$m 0^{59}$. A restrição ao uso desta droga é a necessidade de monitoração hematológica já que a mesma pode induzir agranulocitose. Porém, em estudo recente, Klein et al60 demonstraram que o risco de ocorrência desse tipo de complicação com o uso da clozapina é muito baixo. A quetiapina que apresenta alta afinidade por receptores serotoninérgicos e baixa afinidade por receptores D2, também pode ser utilizada embora seja menos efetiva que a clozapina e eventualmente possa agravar o quadro motor da DP. Em estudo retrospectivo Reddy et al ${ }^{61}$ avaliaram o efeito da quetiapina em pacientes com DP apresentando quadro psicótico definido como presença de delírios e alucinações levando substancial impacto na comunicação e no relacionamento social. Os autores relataram que a quetiapina em dose média de $54 \mathrm{mg}$ por dia foi efetiva para o controle completo ou parcial do quadro psicótico em $81 \%$ dos pacientes e em apenas $13 \%$ dos casos constatou-se piora do quadro motor, sendo que entre este predominavam pacientes com quadro demencial. Existe uma preocupação a respeito do aumento de risco de mortalidade relacionado ao uso de neurolépticos atípicos em pacientes idosos. Portanto, antes de prescrever drogas desta classe é necessário avaliar riscos e benefícios com cautela.
Estudos recentes, embora não controlados e com limitado número de pacientes, têm demonstrado efeitos benéficos do emprego da rivastigmina e do donepezil no tratamento de quadros psicóticos e alucinações induzidas por medicamentos antiparkinsonianos ${ }^{62,63}$.

Embora os transtornos psicóticos sejam os que mais chamam a atenção, não são as únicas formas de complicações psiquiátricas que trazem redução da qualidade de vida para os pacientes com DP. Alterações de humor, aumento de libido e outras distúrbios de comportamento eventualmente estão presentes em indivíduos com DP.

A elevação do humor não ocorre em pacientes com DP não tratada, entretanto, Celesia e Barr ${ }^{64}$ observaram euforia em $10 \%$ dos pacientes tratados com levodopa. Goodwin ${ }^{65}$ relatou uma incidência de $1,5 \%$ de hipomania em 908 pacientes tratados com levodopa e em virtualmente todos aqueles que apresentavam história de mania antes da instalação da DP. Esses quadros quando não respondem à redução da dose do antiparkinsoniano, devem ser tratados com carbamazepina ou valproato.

Pacientes com DP podem apresentar um aumento da libido coincidente com o início do tratamento ou aumento das doses de medicamentos. Geralmente essa melhora da função sexual representa apenas um retorno à atividade sexual normal, decorrente da recuperação do desempenho motor e da sensação de bem estar. Esse ressurgimento do interesse sexual eventualmente pode ser frustrado por impotência sexual, muitas vezes presente em pacientes com DP avançada. Mais raramente, contudo, hipersexualidade e comportamento sexual aberrante podem ser induzidos por agentes dopaminérgicos. Essas alterações geralmente desaparecem com a redução da dose da medicação em uso.

Evans e Lees observaram que uma pequena proporção de pacientes com DP sofre de distúrbios motores e psiquiátricos diretamente atribuídos ao uso excessivo de drogas dopaminérgicas, superior ao recomendado e ao necessário para o tratamento adequado66. No consultório, esses pacientes solicitam aumento de doses de levodopa, reclamam de ineficiência de doses usuais e aumentam doses de medicações por conta própria ignorando conselhos médicos. Esses pacientes aparentam não se importar com os malefícios desta prática e toleram efeitos colaterais de altas doses. Geram assim um comportamento autodestrutivo, semelhantes ao visto em viciados em drogas ilícitas. Esta complicação da terapia é reconhecida como síndrome da desregulação dopaminérgica e não tem terapia especifica com eficácia comprovada ${ }^{66}$.

Uso de agonistas dopaminérgicos parece provocar o vício do jogo patológico em população suscetível. Os fatores de risco encontrados para esse comportamen- 
to foram: início de DP em idade mais jovem e história familiar ou pessoal de abuso de álcool ${ }^{67,68}$. A dopamina é o neurotransmissor responsável pela sensação de recompensa, e provavelmente seja essa uma das causas da síndrome da desregulação dopaminérgica e jogo patológico.

\section{Distúrbios do Sono}

Cerca de $70 \%$ do pacientes com DP apresentam transtornos do sono e cerca de 15\% têm sonolência diurna excessiva. Esses fatores provocam complicações como cansaço, fadiga, irritabilidade e, portanto, comprometem a qualidade de vida dos parkinsonianos 69,70 . Deve ser notado que sonolência excessiva pode até mesmo provocar acidentes de carro, como demonstraram Hobson et al ${ }^{17}, 3,8 \%$ de um grupo de pacientes com DP estudados quanto a este aspecto relatavam episódios de sono incontrolável dirigiam.

Os distúrbios do sono tornam-se mais freqüentes em fases mais avançadas da DP, possivelmente porque a doença torna-se mais grave e assim aumentam as complicações (motoras ou não), além do que o avanço da doença é acompanhado por aumento de doses de medicações e em paralelo, de seus efeitos colaterais ${ }^{71}$. Soma-se a isso o fato de que o próprio envelhecimento provoca alterações na arquitetura de sono e do ciclo circadiano.

As disfunções motoras provocada pela DP podem trazer prejuízo ao sono pois a bradicinesia pode impedir que paciente mude de posição na cama durante a noite. A presença de tremor e rigidez também podem comprometer o conforto, assim como distonia e discinesias provocadas pelo tratamento. Porém outras complicações como as psiquiátricas (alucinações, ilusões, demência, sonhos vívidos, pesadelos, depressão, ataques de pânico), as autonômicas (noctúria), dor e efeito adverso de drogas podem contribuir para alterações do sono.

Distúrbio comportamental da fase de movimentos oculares rápidos do sono (fase REM), síndrome das pernas inquietas e movimentos periódicos dos membros são mais freqüentes na DP do que na população em geral e parecem estar relacionados às alterações neurobiológicas da DP. Insônia e sono fragmentado são usuais na DP e provavelmente dependem de distúrbio primário de sono embutido na progressão da doença ou desenvolvem-se como complicação da terapia ${ }^{72}$.

O distúrbio comportamental do sono REM é caracterizado por movimentos bruscos durante o sono REM que por vezes pode causam incômodo ou mesmo ferimentos ao cônjuge ou ao próprio paciente, e por atividade motora durante período de sono que deveria corresponder à atonia da fase REM. É um distúrbio freqüente e compromete cerca de $20 \%$ dos pacientes com
$\mathrm{DP}^{69}$. O tratamento é simples e se baseia em pequenas doses de clonazepam.

Embora alguns autores admitam uma relação entre síndrome das pernas inquietas e DP, ainda existem controvérsias sobre esse tema ${ }^{73}$. A síndrome das pernas inquietas pode estar correlacionada com neuropatia periférica, anemia, e geralmente é tratada com agonistas dopaminérgicos ou levodopa, administrados na hora de dormir.

\section{Drogas como selegilina e amantadina e altas doses de levodopa podem causar insônia.}

Sonolência diurna excessiva tem etiologia multifatorial. Dificuldade em manter o sono ou em iniciá-lo, decorrente das possíveis alterações relacionadas à DP, podem ser uma das causas e o efeito residual de sedativos (como benzodiazepinicos e antidepressivos) é capaz de proporcionar sonolência diurna. Todos os agentes dopaminérgicos podem potencializar a sonolência excessiva. Alguns dos afetados têm apenas sonolência enquanto que outros sofrem de ataques de sono incontroláveis. Paus et al ${ }^{74}$ estimaram que cerca de $1 \%$ dos pacientes com DP podem sofrer desses ataques, sem sinais de alerta prévio ou sonolência precedente. Arnulf et $\mathrm{a}^{75}$ sugerem que sono excessivo e ataques repentinos de sono podem ser gerados pela alterações neurobiológicas próprias da DP.

\section{Ansiedade e Transtorno Obssessivo-Compulsivo na Doença de Parkinson}

A prevalência de ansiedade na DP varia entre 5\% a $40 \%$ e é maior que na população em geral ${ }^{76,77}$. Eventualmente a ansiedade pode apresentar-se sob formas graves como ataques de pânico, ansiedade generalizada e distúrbios fóbicos. Sintomas somáticos como falta de ar, desconforto toráxico, dor abdominal e tonturas, entre outros, podem ocorrer, assim como medo incontrolável de institucionalização, de perder a sanidade mental ou de morrer. A causa de ansiedade na DP ainda não foi definida mas parece ser uma reação psicológica à doença embora não se possa descartar a possibilidade de estar relacionada a modificações neuroquímicas causadas pelo processo degenerativo da DP, que leva a uma redução progressiva de dopamina, serotonina, e noradrenalina.

É fato bastante conhecido que a ansiedade é um fator precipitante de piora das manifestações motoras da DP, particularmente do tremor. O tratamento da ansiedade na DP, quando necessário é feito através do emprego dos ansiolíticos usuais, com a ressalva de que essas drogas podem piorar a instabilidade postural e a confusão mental, caso presentes. Pelo fato de haver coexistência de ansiedade e depressão, frente a um paciente com ansiedade, depressão devera ser pron- 
tamente investigada. O emprego de antidepressivos pode reduzir os níveis de ansiedade.

Comportamentos repetitivos têm sido descritos sob diversos diagnósticos na DP. O transtorno obssessivocompulsivo (TOC) pode ser observado em diversas doenças dos núcleos da base, inclusive na DP. A literatura sobre sintomas obssessivos-compulsivos na DP é contraditória. Alguns estudos evidenciam associação positiva com DP e outros mostram resultado contrário $^{78,79}$. Em pacientes com DP que apresentam TOC, o tratamento segue as mesmas linhas terapêuticas utilizadas para o TOC primário.

\section{Distúrbios autonômicos}

Embora parkinsonismo associado a alterações autonômicas sugira diagnóstico de atrofia de múltiplos sistemas, pacientes com DP comumente apresentam algum tipo comprometimento autonômico, sendo que essas alterações tornam-se mais evidentes com o avançar da doença. É importante valorizar a idéia que ao avaliar um paciente com DP e com queixas referentes à disautonomia, doenças não correlacionadas à DP deverão ser afastadas, pois os parkinsonianos geralmente têm idade avançada, período em que comorbidades podem ocorrer.

Vários tipos de transtornos autonômicos podem estar presentes e a seguir descrevemos os mais importantes ${ }^{80}$

a) Hipotensão Ortostática: pode surgir por disfunções autonômicas periféricas ou centrais. A causa é degeneração neuronal e corpos de Lewy acometendo o sistema nervoso autonômico periférico e central. Pacientes com DP e disautonomia frequentemente têm pressão arterial elevada na posição supina, o que pode levar ao diagnóstico errôneo de hipertensão arterial sistêmica. Neste caso o uso de anti-hipertensivos pode piorar os sintomas. Vários medicamentos, incluindo drogas antiparkinsonianas podem também provocar queda de pressão. A avaliação de pacientes com hipertensão ortostática deve levar em consideração todas as medicações em uso. A redução de doses ou a suspensão de certas drogas podem trazer benefícios ao paciente. O tratamento pode conter medidas não farmacológicas como uso de meias elásticas, dormir com cabeceira elevada e aumento de ingesta de sal. Uso de drogas como fludrocortisona e midrodine podem ser considerados, caso as medidas iniciais falhem.

b) Distúrbios do trato gastrointestinal: a constipação é uma queixa comum e pode ser decorrente da DP ou de efeito de medicações como anticolinérgicos. $\mathrm{O}$ tratamento requer afastar efeitos indesejáveis de drogas suspendendo certas medicações, mudança de dieta, exercícios e eventualmente o emprego de laxativos.
Disfagia é outro problema comum, e afeta pacientes com tempo maior de evolução da doença e em idade mais avançada. Atendimento fonoaudiólogo e mudança de consistência de dieta são medidas terapêuticas que auxiliam o tratamento. Em casos avançados a gastrostomia pode ser indicada.

c) Distúrbios urológicos: noctúria tende a ser o primeiro sintoma urológico e o mais comum em pacientes com DP. È seguido por outros como urgência miccional e dificuldade em iniciar micção. Avaliação urológica é importante, pois os sintomas não necessariamente decorrem da DP, e, portanto, moléstias primariamente urológicas devem ser sempre descartadas. Teste urodinâmico pode auxiliar o diagnóstico. Oxibutinina pode ser prescrita como terapêutica.

d) Distúrbios sexuais: dificuldades em iniciar ereção e em mantê-la são problemas comumente relatados por homens afetados pela DP. As mulheres, por sua vez, têm risco de não mais atingir orgasmo. Drogas dopaminérgicas podem aumentar libido e de certa forma, encontram minimizar essa disfunção. Entretanto, existe o risco de causarem hipersexualidade. O uso de sildenafil traz benefícios para impotência em certos casos, quando a administração de agente dopaminérgico falha.

e) Sudorese: alguns parkinsonianos têm sudorese excessiva enquanto que outros, em número menor, sofrem de hipohidrose. Sudorese excessiva pode ocorrer em períodos off, em pacientes com flutuações motoras associadas à terapia dopaminérgica.

\section{Distúrbios da sensibilidade}

Dor acompanha cerca de $50 \%$ dos pacientes com DP e é geralmente inespecífica. Sintomas dolorosos por vezes são associados à distonia de fim de dose de levodopa. É interessante notar que a queixa de dor freqüentemente surge nos períodos off. Sendo assim, otimização de terapêutica antiparkinsoniana faz parte da terapêutica da dor, assim como a atividade física regular.

\section{Flutuações não motoras}

Uma grande parte dos pacientes com DP que estão sob tratamento com levodopa, desenvolvem uma complicação da terapêutica que são as flutuações motoras, como exemplos podem ser citados as discinesias e o fenômeno wearing-off (deterioração de fim de dose) Foi estimado que cerca de dois terços dos pacientes com flutuações motoras sofrem também de flutuações no humor ${ }^{81}$. Essas modificações do humor podem acontecer várias vezes ao dia, variando de deprimido/ suícida para eufórico e hipomaniaco. Essas flutuações 
podem ser tão incapacitantes quanto às flutuações motoras $^{82}$. Richard et al ${ }^{83}$ demonstraram que flutuações de humor podem surgir em indivíduos sem flutuações motoras e que ansiedade pode surgir nos períodos off, coincidindo freqüentemente (mas nem sempre) com piora de humor.

Witjas et $\mathrm{al}^{84}$ demonstraram que todos os pacientes que participaram do estudo e que sofriam flutuações motoras, também lidavam com flutuações não mo- toras. Neste estudo, ansiedade, sudorese excessiva, lentificação do pensamento, fadiga e acatisia foram os mais proeminentes sintomas de flutuações não motoras. Stacy et al ${ }^{85}$ também demonstraram a importância das flutuações não motoras, ao verificar que manifestações não motoras podem predominar em relação às motoras. Alguns sintomas de wearing off pouco estudados também comprometem a vida dos parkinsonianos como dificuldade em iniciar micção e dor.
Quadro 1 Doença de Parkinson Manifestações Neuropsiquiátricas

- Alterações Cognitivas / Demência

- Depressão

- Distúrbios do Sono

- Alucinações / Delírio / Delirium

- Mania / Hipomania

- Hipersexualidade

- Ansiedade / Crises de Pânico

- Transtorno Obsessivo Compulsivo
Quadro 2-Alterações Psiquiátricas Relacionadas a Drogas Antiparkinsonianas

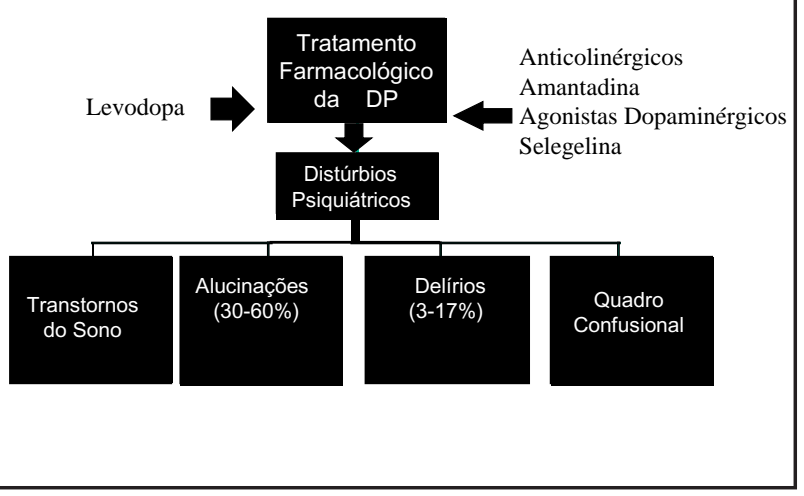

\section{Quadro 3: Condutas em Distúrbios \\ Psiquiátricos Relacionadas a Drogas Antiparkinsonianas}

Retirar Anticolinérgicos, Amantadina, Selegelina e Agonistas Dopaminérgicos

Diminuir Levodopa

Associar Neurolépticos Atípicos: Quetiapina, Clozapina e Olanzapina 


\section{REFERÊNCIAS BIBLIOGRÁFICAS}

1. Bronnick K, Aarsland D, Larsen JP. Neuropsychiatric disturbances in Parkinson's disease clusters in five groups with different prevalence of dementia. Acta Psychiatr Scand 2005;112: 201-207.

2.Weintraub D, Moberg PJ, Duda JE, Katz IR, Stern MB. Effect of Psychiatric and Other Nonmotor Symptoms on Disability in Parkinson's Disease. J Am Geriatr Soc 2004;52:784-788.

3.Chaudhuri KR, Martinez-Martin P, Schapira AH, Stocchi F, Sethi K, Odin P, et al. ABN abstracts: The Association of British Neurologists Autumn Meeting, London, UK, 4-6 October 2006: Posters International validation of the first non motor questionnaire (NMSQuest) and scale (NMSS) for Parkinson's disease. J Neurol Neurosurg Psychiatr 2007; 78:213.

4.Shulman LM, Taback RL, Bean J, Weiner WJ. Comorbidity of the nonmotor symptoms of Parkinson's disease. Mov Disord 2001;16:507-510.

5. McKeith IG, Dickson DW, Lowe J, Emre M, O'Brien JT, Feldman H, et al. Diagnosis and management of dementia with Lewy bodies: third report of the DLB Consortium. Neurology 2005; 65:1863-1872.

6. de Lau LM, Schipper CM, Hofman A, Koudstaal PJ, Breteler MM. Prognosis of Parkinson disease: risk of dementia and mortality: the Rotterdam Study. Arch Neurol 2005; 62:1265-1269.

7. Schrag A, Jahanshahi M, Quinn N. What contributes to quality of life in patients with Parkinson's disease? J Neurol Neurosurg Psychiatr 2000; 69:308-312.

8. Barbosa ER, Lefevre BH, Comerlatti LR, Scaff M, Canelas HM. Disfunções neuropsicológicas na doença de Parkinson. Estudo de 64 casos. Arq Neuropsiquiatr 1986; 44:55-59

9. Aarsland D, Andersen K, Larsen JP, Lolk A, Kragh-Sorensen P. Prevalence and characteristics of dementia in Parkinson's disease: an 8-year prospective study. Arch Neurol 2003; 60:387-392.

10. Aarsland D, Bronnick K, Ehrt U, De Deyn PP, Tekin S, Emre M, et al. Neuropsychiatric symptoms in patients with Parkinson's disease and dementia: frequency, profile and associated care giver stress. J Neurol Neurosurg Psychiatry 2007;78:36-42.

11. Pluck CG, Brow RG. Apathy in Parkinson's disease J. Neurol. Neurosurg. Psychiatry 2002; 73;636-642.

12.Kirsch-Darrow L, Fernandez HF, Marsiske M, Okun MS. Dissociating apathy and depression in Parkinson disease. Neurology 2006;67:33-38.

13.Marder $\mathrm{K}$, Cote $\mathrm{L}$, Tang $\mathrm{M}$. The risk and predictive factors associated with dementia in Parkinson's disease. In: Korczyn A. Dementia in Parkinson's Disease. Bologna: Monduzzi, 1994, p 51-54.

14. Janvin CC, Larsen JP, Aarsland D, Hugdahl K. Subtypes of mild cognitive impairment in Parkinson's disease: Progression to dementia. Mov Disord 2006; 21:1343-1349.

15. Huang X, Chen P, Kaufer DI, Troster Al, Poole C. Apolipoprotein E and dementia in Parkinson disease: a meta-analysis. Arch Neurol 2006; 63:189-193.

16. Aarsland D, Andersen K, Larsen JP, Lolk A, Nielsen H, Kragh-Sorensen P. Risk of dementia in Parkinson's disease: A community-based, prospective study. Neurology 2001; 56:730-736

17. Hobson DE, Lang AE, Martin WR, Razmy A, Rivest J, Fleming J. Excessive daytime sleepiness and sudden-onset sleep in Parkinson disease. A survey by the Canadian Movement Disorders Group. JAMA 2002;287:455-463.

18,Woods SP, Troster Al. Prodromal frontal/executive dysfunction predicts incident dementia in Parkinson's disease. J Int Neuropsychol Soc 2003;9:17-24.

19.Emre M. Dementia associated with Parkinson's disease. Lancet Neurol 2003;2:229-237.

20. Miyasaki JM, Shannon K, Voon V, Ravina B, Kleiner-Fisman G, Anderson K, et al. Practice Parameter: evaluation and treatment of depression, psychosis, and dementia in Parkinson disease (an evidence-based review): report of the Quality Standards Subcommittee of the American Academy of Neurology. Neurology 2006;66:996-1002.

21. Marinus J, Visser M, Verwey NA, Verhey FR, Middelkoop HA, Stiggelbout AM, et al. Assessment of cognition in Parkinson's disease. Neurology. 2003; 61:1222-1228

22.Mahieux F, Michelet D, Manifacier M, Boller F, Fermanian J, Guillard A. Minimental Parkinson: first validation study of a bedside test constructed for Parkinson's disease. Behav Neurol 1995;8:15-22.

23. Klatka LA, Louis ED, Schiffer RB. Psychiatric features in diffuse Lewy body disease: A clinicopathologic study using Alzheimer's disease and Parkinson's disease comparison groups. Neurology 1996;47:1148-1152.

24.Bullock R, Cameron A. Rivastigmine for the treatment of dementia and visual hallucinations associated with Parkinson's disease. Curr Med Res Opin 2002; 18(5):258-264

25. Ravina B, Putt M, Siderowf A, Farrar JT, Gillespie M, Crawley A, et al. Donepezil for dementia in Parkinson's disease: a randomised, double blind, placebo controlled, crossover study J Neurol Neurosurg Psychiatr
2005;76:934-939.

26. Aarsland D, Andersen K, Larsen JP, Lolk A, Kragh-Sorensen P. Prevalence and characteristics of dementia in Parkinson's disease: a 8-year prospective study. Arch Neurol 2003;60:387-392.

27.Emre M, Aarsland D, Albanese A, Byrne EJ, Deuschl G, De Deyn PP, et al. Rivastigmine for dementia associated with Parkinson's disease. N Engl J Med 2004;351:2509-2518.

28. Poewe W, Wolters E, Emre M, Onofri M, Hsu C, Tekin S, et al. Long-term benefits of rivastigmine in dementia associated with Parkinson's disease: an active treatment extension study. Mov Disord. 2006;21:456-561

29.Prado RCF, Barbosa ER. Depression in Parkinson's Disease. Arq Neuropsiquiatr 2005;63:766-771

30. Kostic VS, Filipovic SR, Lecic D, Momcilovic D, Sokic D, Sternic N. Effect of age at onset on frequency of depression in Parkinson's disease. J Neuro Neurosurg Psychiatr 1994;57:1265-1257.

31 Starkstein SE, Petracca G, Chemerinski E, Teson A, Sabe L, Merello M, et al. Depression in classic versus akinetic-rigid Parkinson's disease. Mov Disord 1998;13:29-33

32. Rojo A, Aguilar M, Garolera MT, Cubo E, Navas I, Quintana S. Depression in Parkinson's disease: clinical correlates and outcome Parkinsonism Relat Disord 2003;10:23-28.

33. Starkstein SE, Preziosi TJ, Bolduc PL, Robinson RG. Depression in Parkinson's disease. J N Ment Dis 1990;178:27-31.

34. McDonald WM. Richard IH, Delong MR. Prevalence, etiology, and treatment of depression in Parkinson's disease. Biol Psychiatry 2003;54:363-375.

35.Lieberman A. Depression in Parkinson's disease - a review. Acta Neuro Scand 2006;113:1-8

36. Choi $\mathrm{CH}$, Sohn YH, Kim JS. Depression in patients with Parkinson's disease: A follow-up comparative study after long-term levodopa therapy. Mov Disord 1997;12(Suppl 1):125.

37.Barone P, Scarzella L, Marconi R, Antonini A, Morgante L, Bracco F, et al and the Depression/Parkinson Italian Study Group. Pramipexole versus sertraline in the treatment of depression in Parkinson's disease. A national multicenter parallel-group randomized study. J Neurol 2006;253:601-607.

38. Weintraub D, Morales KH, Moberg PJ, Bilker WB, Balderston C, Duda JE, et al. Antidepressant studies in Parkinson's disease: a review and metanalysis. Mov Disord 2005; 20:1161-1169.

39. Richard IH. Do serotonin reuptake inhibitor antidepressants worsen Parkinson's disease? A retrospective case series. Mov Disord 1999;14:155-163.

40.Okun MS, Watts RL. Depression in Parkinson's disease. Neurology 2002;58: S63-S70

41.Fregni F, Santos CM, Myczkowski ML, Rigolino R, Gallucci-Neto J, Barbosa ER, et al. Repetitive transcranial magnetic stimulation is as effective as fluoxetine in the treatment of depression in patients with Parkinson's disease. J Neurol Neurosurg Psychiatr 2004; 75:1171-1174

42. Voon V, Saint-Cyr JA, Lozano AM, Moro E, Dujardin K, Lang AE, et al. Suicide risk in patients with Parkinson's disease undergoing subthalamic stimulation. Mov Disord. 2004;19(suppl9):S410-S411.

43.Burckard PR, Vingerhoets A, Berney A, Bogousslavsky J, Villemure JG, Ghika J. Death by suicide after deep brain stimulation. Mov Disord 2004;19(Suppl 9):S316

44.Martinez-Martin P, Catalan MJ, Benito-Leon J, Moreno AO, Zamarbide I, Cubo $E$, et al. Impact of fatigue in Parkinson's disease: the Fatigue Impact Scale for Daily Use (D-FIS). Qual Life Res 2006;15(4):597-606.

45.Alves G, Wentzel-Larsen T, Larsen JP. Is fatigue an independent and persistent symptom in patients with Parkinson disease? Neurology 2004;63:19081911.

46. Herlofson K, Larsen JP. The influence of fatigue on health-related quality of life in patients with Parkinson's disease Acta Neurol Scand 2003:107:1-6.

47. Lou JS, Kearns G, Oken B, Sexton G, Nutt J. Exacerbates physical fatigue and mental fatigue in Parkinson's disease. Mov Disord 2001;16:190-196.

48. Oved D, Ziv I, Treves TA, Paleacu D, Melamed E, Djaldetti R Effect of dopamine agonists on fatigue and somnolence in Parkinson's disease. Mov Disord 2006:8:1257-1261.

49.Lou JS, Kearns G, Benice T, Oken B, Sexton G, Nutt J Levodopa improves physical fatigue in Parkinson's disease: A double-blind, placebo-controlled, crossover study Mov Disord 2003; 10:1108-1114.

50.Fenelon G, MahieuX F, Huon R, Ziégler M. Hallutinations in Parkinson's disease: prevalence, phenomenology and risk factors. Brain 2000; 123:733-745.

51.Goetz CG, Wuu J, Curgian LM, Leurgans S. Hallucinations and sleep disorders in PD. Six-year prospective longitudinal study. Neurology 2005;64:81-86.

52.Graham JM, Grunwald RA, Sagar HJ. Hallucinations in idiopathic Parkinson's 
disease. J Neurol Neurosurg Psychiatr 1997;63:434-440.

53.Barbosa ER, Limongi JCP, Cummings J. Parkinson's disease. Psychiat Clin 1997:20:769-790.

54. Goetz CG, Pappert EJ, Blasucci LM, Stebbins GT, Ling ZD, Nora MV, et al. Intravenous levodopa in hallucinating Parkinson's disease patients: high dose challenge does not precipitate hallucinations. Neurology 1998;50:515-517

55.Sanchez-Ramos JR, Ortoll R, Paulson GW. Visual hallucinations associated with Parkinson disease. Arch Neurol 1996;53:1265-1268

56.Fenelon G, Goetz CG, Karenberg A Hallucinations in Parkinson disease in the prelevodopa era. Neurology 2006;66:93-98

57.Poewe WH, Deuschl G, Gordin A, Kultalahti ER, Leinonen M, The Celomen Study Group. Efficacy and safety of entacapone in Parkinson's disease patients with suboptimal levodopa response: a 6-month randomised placebocontrolled double-blind study in Germany and Austria (Celomen study). Acta Neurol Scand 2002;105:245-255.

58.Juncos JL. Management of psychotic aspects of Parkinson's disease. J Clin Psychiatr 1999;60(Suppl 8):42-53.

59. Pollak P, Tison F, Rascol O, Destee A, Pere JJ, Senard JM, et al. Clozapine in drug induced psychosis in Parkinson's disease: a randomized, placebo-controlled study with open follow up. J Neurol Neurosurg Psychiatr 2004; 74:689-695.

60.Klein C, Gordon J, Pollak L, Rabey JM. Clozapine in Parkinson's disease psychosis: 5-year follow-up review. Clin Neuropharmacol 2003, 26:8-11.

61.Reddy S, Factor SA, Molho ES, Feustel PJ. The effect of quetiapine on psychosis and motor function in parkinsonian patients with and without dementia. Mov Disord 2002;17:676-681.

62.Bergman J, Lerner V. Successful use of donepezil for the treatment of psychotic symptoms in patients with Parkinson's disease. Clin Pharmacol 2002;25:107-110.

63.Aarsland D, Hutchinson M, Larsen JP. Cognitive, psychiatric and motor response to galantamine in Parkinson's disease with dementia. Int J Geriat Psychiatr 2003; 18(10):937-941.

64.Celesia GG, Barr AN. Psychosis and other psychiatric manifestations of levodopa therapy. Arch Neurol 1970;23(3):193-200.

65.Goodwin FK. Psychiatric side effects of levodopa in man. JAMA 1971;218(13):1915-1920.

66. Evans AH, Lees AJ. Dopamine dysregulation syndrome in Parkinson's disease Current Opinion in Neurology 2004; 17:393-398

67. Voon V, Thomsen T, Miyasaki JM, de Souza M, Shafro A, Fox SH, et al. Factors Associated With Dopaminergic Drug-Related Pathological Gambling in Parkinson DiseaseArch Neurol 2007:64:212-216.

68. Voon V, Hassan K, Zurowski M, Duff-Canning S. Prospective prevalence of pathologic gambling and medication association in Parkinson disease. Neurology 2006;66:1750-1752.

69.Askenasy JJ. Sleep in Parkinson's Disease. Acta Neurol Scand 1993;
$87: 167-170$

70.Tandberg E, Larsen JP, Karlsen K. Excessive daytime sleepiness and sleep benefit in Parkinson's disease: a community-based study. Mov Disord 1999:14:922-927.

71.Kumar S, Bhatia M, Behari M. Sleep disorders in Parkinson's disease. Mov Disord 2002:7:775-781.

72. Brunner $\mathrm{H}$, Wetter TC, Hogl B, Yassouridis A, Trenkwalder C, Friess E. Microstructure of the non-rapid eye movement sleep electroencephalogram in patients with newly diagnosed Parkinson's disease: effects of dopaminergic treatment. Mov Disord 2002;17:928-993.

73.Adler CH, Thorpy MJ. Sleep issues in Parkinson's disease. Neurology 2005;64: S12-S20.

74. Paus S, Brecht HM, Koster J, Seeger G, Klockgether T, Wullner U. Sleep attacks, daytime sleepiness, and dopamine agonists in Parkinson' disease. Mov Disord 2003;18:659-667.

75.Arnulf I, Konofal E, Merino-Andreu M, Houeto JL, Mesnage V, Welter ML, Houeto JL, Mesnage V, Welter ML, et al. Parkinson's disease and sleepiness - an integral part of PD. Neurology 2002;58:1019-1024.

76.Walsh K, Bennett G. Parkinson's disease and anxiety Postgrad. Med $J$ 2001;77:89-93.

77.Schiffer RB, Kurlan R, Rubin A, Boer S. Evidence for atypical depression in Parkinson's disease. Am J Psychiatry 1988;145(8):1020-1022.

78. Maia AF, Pinto AS, Barbosa ER, Menezes PR, Miguel EC. Obssessive compulsive symptoms, obssessive compulsive disorder and related disorders in Parkinson's disease. J Neuropsychiatr Clin Neurosci 2003;15:371-374.

79.Kurlan R. Repetitive behaviors in Parkinson's disease. Mov Disord 2004;19: 433-469.

80. Olanow WC, Watts RL, William C, Koller WC. An algorithm (decision tree) for themanagement of Parkinson'sdisease (2001). Treat Guidelines 2001;56: S1-S88.

81. Nissenbaum H, Quinn NP, Brown RG, Toone B, Gotham AM, Marsden CD. Mood swings associated with the "on-off " phenomenon in Parkinson's disease. Psychol Med 1987;17:899-904.

82.Cantello R, Gilli M, Riccio A, Bergamasco B. Mood changes associated with "endof-dose deterioration" in Parkinson's disease: a controlled study. J Neurol Neurosurg Psychiatr 1986;49:1182-1190

83. Richard IH, Samuel Frank S, McDermott MP, Wang H, Justus AW, Ladonna KA, et al. The Ups and Downs of Parkinson Disease A Prospective Study of Mood and Anxiety Fluctuations. Cogn Behav Neurol 2004;17:201-207.

84.Witjas T, Kaphan E, Azulay JP, Blin O, Ceccaldi M, Pouget J, et al. Nonmotor fluctuations in Parkinson's disease: frequent and disabling. Neurology 2002:59:408-413.

85.Stacy M, Bowron A, Guttman M, Hauser R, Hauser R, Hughes K, et al. Identification of Motor and nonmotor Wearing-off in Parkinson's Disease: Comparison of a patient Questionnaire versus a Clinician assessment. Mov disord 2005;20:726-733. 